\title{
Análise Retrospectiva de Cirurgias Rinossinusais em um Hospital Escola
}

\section{Sinus Retrospective Analysis of Surgeries in a Hospital School}

\author{
Thiago Bittencourt Ottoni de Carvalho*, Tiago José Conrado**, Tiago L Genaro**, \\ Atílio Maximino Fernandes ***, José Victor Maniglia****.
}

\author{
* Médico. Residente ORL. \\ ** Acadêmico de Medicina da Faculdade de Medicina de São José do Rio Preto - FAMERP - SP. \\ **** Doutor. Médico Assistente do Departamento de Otorrinolaringologia e Cirurgia de Cabeça e Pescoço da Faculdade de Medicina de São José do Rio Preto - FAMERP \\ $-\mathrm{SP}$. \\ **** Livre Docente.Médico Assistente do Departamento de Otorrinolaringologia e Cirurgia de Cabeça e Pescoço da Faculdade de Medicina de São José do Rio Preto - \\ FAMERP - SP) \\ Instituição: Departamento de Otorrinolaringologia e Cirurgia de Cabeça e Pescoço da Faculdade de Medicina de São José do Rio Preto - FAMERP - SP. \\ São José do Rio Preto / SP - Brasil. \\ Endereço para correspondência: Thiago Bittencourt Ottoni de Carvalho - Rua José Picerni 449 - Apto 21 - Jardim Panorama - São José do Rio Preto / SP - Brasil - CEP: \\ 15091-200 - Telefone: (+55 17) 8141-5584/(+55 61) 7814-7648 - E-mail: drthiago.ottoni@yahoo.com.br \\ Artigo recebido em 23 de Julho de 2010. Artigo aprovado em 22 de Agosto de 2010.
}

\section{RESUMO}

Introdução:

A otorrinolaringologia é uma especialidade médica que abrange 4,4\% do total de médicos paulistas, tendo uma variedade de procedimentos cirúrgicos distribuídos entre faringe, nariz, ouvido e laringe. O conhecimento do perfil de um serviço de referência em otorrinolaringologia permite a sua melhor organização, dimensionamento do volume do atendimento e das cirurgias realizadas, proporcionando uma melhor formação ao aluno e ao médico residente.

Objetivo: Descrever o perfil das cirurgias nasossinusais e dos pacientes submetidos a elas no departamento de otorrinolaringologia e cirurgia de cabeça e pescoço de um hospital escola.

Método: Foi realizado estudo de coorte histórico com corte transversal retrospectivo, com revisão de 872 prontuários de pacientes submetidos a cirurgias rinossinusais entre janeiro de 2006 e dezembro de 2008. Utilizado questionário próprio, buscando sexo, idade, diagnóstico cirúrgico e cirurgia realizada.

Resultados: Do total de 872 pacientes analisados, 45,4\% eram sexo feminino e 54,6\% sexo masculino, variando em uma faixa etária 4 a 80 anos (média de 29,8 anos). Os principais diagnósticos cirúrgicos foram: desvio septo nasal(n=457), deformidade nasal pós-trauma $(n=287)$, hipertrofia de conchas nasais $(n=153)$, polipose rinossinusal $(n=73)$, rinossinusite crônica $(n=32)$. Entre os procedimentos cirúrgicos mais frequentemente realizadas, estão: septoplastia $(n=388)$, rinosseptoplastia $(n=215)$, FESS $(n=131)$, cauterização intra-turbinal $(n=114)$, rinoplastia reparadora( $n=73)$, turbinectomia( $n=43)$, turbinoplastia $(n=55)$. Ressalta-se que os pacientes podem ter recebido mais de um diagnóstico cirúrgico e realizado mais de uma cirurgia, dependendo da indicação.

Conclusão: Apresenta-se o volume e a diversidade de cirurgias rinossinusais realizadas em nosso serviço, contribuindo com a escassa produção científica sobre esse tipo de casuística.

Palavias-chave: doenças nasais, nariz, neoplasias nasais, rinoplastia, procedimentos cirúrgicos otorrinolaringológicos.

\section{SUMMARY}

Introduction:

The ENT is a medical specialty that covers $4.4 \%$ of all doctors in São Paulo, with a variety of surgical procedures distributed between pharynx, nose, ear and larynx. Knowing the profile of a reference service in otolaryngology allows for a better organization, scaling the volume of care and surgeries, providing better training to the student and resident physician.

Objective: To describe the profile of sinonasal surgery and patients to them in the department of otolaryngology and head and neck surgery at a teaching hospital.

Method: We conducted a cohort study of cross-sectional retrospective study with review of 872 charts of patients undergoing surgery Sinus between January 2006 and December 2008. Used questionnaires, seeking sex, age, surgical diagnosis and surgery.

Results:

Of 872 patients studied, $45.4 \%$ were female and 54.6\% male, ranging in an age group 40-80 years (mean 29.8 years). The main surgical diagnoses were: nasal septum deviation $(n=457)$, nasal deformity after trauma ( $n=287)$, enlarged turbinates $(n=153)$, rhinosinusal polyposis $(n=73)$, chronic sinusitis $(n=32)$. Among the most frequently performed surgical procedures include: septoplasty $(n=388)$, rhinoplasty $(n=215)$, FESS $(n=131)$, intra-turbinal cauterization $(n=114)$, reconstructive rhinoplasty $(n=73)$, turbinectomy $(n=43)$, turbinoplasty ( $n=55)$. It is emphasized that patients may have received more than one surgical diagnosis and realized more than one surgery, depending on the alert.

Conclusion: We present the volume and diversity of Sinus surgeries performed in our department, contributing to the scarce scientific literature on this type of case.

Keywords: nose diseases, nose, nose neoplasms; rhinoplasty; otorhinolaryngologic surgical procedures 


\section{INTRODUÇÃO}

A otorrinolaringologia é a especialidade médica que abrange $4,4 \%$ dos médicos paulistas (1), com 2450 profissionais atuando no estado de São Paulo, de acordo com o censo 2007-2008 realizado pela Associação Brasileira de Otorrinolaringologia e Cirurgia de Cervico-Facial (2). No país, em 2003, pelo Sistema Único de Saúde, foram realizados 80.030 procedimentos cirúrgicos otorrinolaringológicos (3). Destes, $45,6 \%$ foram na faringe; $28 \%$ no nariz; $14 \%$ no ouvido; e $12,4 \%$ na laringe. Entre as cirurgias rinossinusais, $60 \%$ foram realizadas em homens e $40 \%$ em mulheres. A distribuição por idade desse tipo de cirurgia apresenta-se com a seguinte ocorrência: 1,2\% do total ocorreram abaixo de 4 anos; $2,7 \%$ entre 5 e 9 anos; $23,5 \%$ entre 10 e 19 anos; $51 \%$ entre 20 e 39 anos; 16,8\% entre 40 e 59 anos; e 4,8\% acima de 60 anos, revelando, assim, um perfil masculino, adulto e jovem dos pacientes submetidos a cirurgias rinossinusais no país.

Na clínica privada, por outro lado, as cirurgias rinossinusais são os procedimentos cirúrgicos mais realizados, seguidos pelos procedimentos realizados na faringe (3).

O conhecimento do perfil de um serviço de referência em otorrinolaringologia permite melhor organização do serviço. Além disso, quando se trata de um hospital-escola, o dimensionamento e conhecimento do volume do atendimento e das cirurgias realizadas proporciona uma melhor caracterização e consequente melhor formação ao aluno e ao médico residente.

Este trabalho tem por objetivo descrever o perfil das cirurgias rinossinusais e dos pacientes submetidos a elas peloSUS, no serviço de otorrinolaringologia do Hospital de Base de São José do Rio Preto / SP.

\section{MÉTODO}

Foi realizado estudo de coorte histórico com corte transversal, com revisão de 872 prontuários de pacientes submetidos a cirurgias rinossinusais, entre 2006 e 2008, pelo Departamento de Otorrinolaringologia e Cirurgia de Cabeça e Pescoço da Faculdade de Medicina de São José do Rio Preto, SP - FAMERP.

Os prontuários foram selecionados por meio de busca através da central de processamento de dados (CPD) do Hospital de Base. Foram utilizadas palavras-chave para identificar as cirurgias rinossinusais praticadas no período entre 2006 e 2008, como: "septoplastia", "rinosseptoplastia", "FESS", "cauterização intra-turbinal", "turbinectomia", "turbinoplastia", "cirurgia de Caldwell-Luc", "cirurgia para epistaxe", entre outras.

Foi utilizado um questionário próprio nos prontuários determinados, caracterizando sexo, idade, diagnóstico cirúrgico e cirurgia realizada. Não houve critérios de exclusão. Todas as cirurgias nasossinusais realizadas foram adicionadas ao estudo, no entanto, apenas as principais foram descritas com detalhes, para facilitar a compreensão por parte do leitor.

O projeto de pesquisa foi submetido ao Comitê de Ética em Pesquisa desta instituição e aprovado conforme protocolo número 6209/2007.

\section{RESULTADOS}

Do total de 872 pacientes analisados, $45,4 \%$ foram do sexo feminino e $54,6 \%$ do sexo masculino. A média de idade foi de 29,8 anos, variando em uma faixa etária de 4 a 80 anos. Desse total, 625 pacientes $(69,4 \%)$ tiveram uma única indicação cirúrgica, enquanto $247(30,6 \%)$ apresentaram mais de uma indicação cirúrgica nasal (Tabelas 1 e 2).

Os principais diagnósticos cirúrgicos foram: I) desvio de septo nasal, com 457 pacientes, média de idade de 28,1 anos (5-70 anos), dos quais 39,4\% eram mulheres e 60,6\%, homens; II) deformidade nasal pós-trauma (DNPT), com 287 pacientes, média de idade de 26,7 anos (14-65 anos), dos quais $65,5 \%$ eram mulheres e $34,5 \%$, homens; III) hipertrofia de conchas nasais, com 153 pacientes, média de idade de 28,5 anos (8-69 anos), dos quais 37,3\% eram mulheres e $62,7 \%$, homens; IV) polipose rinossinusal, com 73 pacientes, média de idade de 41,3 anos (9-67 anos), dos quais $34,3 \%$ eram mulheres e $65,7 \%$, homens; V) rinossinusite crônica, com 32 pacientes, média de idade de

Tabela I. Distribuição por sexo.

\begin{tabular}{lcc}
\hline Sexo & $N$ & $\%$ \\
\hline Masculino & 476 & 54,6 \\
Feminino & 396 & 45,4 \\
\hline
\end{tabular}

Tabela 2. Distribuição por faixa etária.

\begin{tabular}{lccc}
\hline Faixa Etária & $\begin{array}{c}\text { Número } \\
\text { de pacientes }\end{array}$ & $\begin{array}{c}\text { Sexo } \\
\text { Masculino }\end{array}$ & $\begin{array}{c}\text { Sexo } \\
\text { Feminino }\end{array}$ \\
\hline $0-9$ anos & $22(2,5 \%)$ & 16 & 6 \\
$10-19$ anos & $195(22,3 \%)$ & 121 & 74 \\
$20-39$ anos & $447(51,3 \%)$ & 213 & 234 \\
$40-59$ anos & $175(20,1 \%)$ & 102 & 73 \\
60 anos ou mais & $33(3,8 \%)$ & 24 & 9 \\
\hline
\end{tabular}


30 anos (4-66 anos), dos quais 37,5\% eram mulheres e 62,5\%, homens (Tabela 3).

Foram realizados 1139 procedimentos cirúrgicos nesse período, sendo que em 631 pacientes as cirurgias foram isoladas (72,3\%) e em 241 (27,7\%) houve uma ou mais cirurgia nasal associada. No mesmo período foram realizados 64.875 atendimentos ambulatoriais, apresentando uma relação de 1,75\% de cirurgias nasossinusais do total de atendimentos (Tabela 4).

Houve prevalência dos seguintes procedimentos: I) septoplastia, em 388 pacientes, média de idade de 28,2 anos (5-70 anos), dos quais 34,8\% eram mulheres e 65,2\%, homens; II) rinosseptoplastia, em 215 pacientes, média de idade de 27,1 anos (14-61 anos), dos quais 62,8\% eram mulheres e 37,2\%, homens; III) cirurgia endoscópica funcional dos seios paranasais (FESS), em 131 pacientes, média de idade de 37,3 anos (4-70 anos), dos quais 37,5\% eram mulheres e 62,5\%, homens; IV) cauterização intraturbinal, em 114 pacientes, média de idade de 26,3 anos (10-61 anos), dos quais 28\% eram mulheres e 72\%, homens; V) rinoplastia reparadora, em 73 pacientes, média de idade de 26,6 anos (17-65 anos), dos quais 76,7\% eram mulheres e 23,3\%, homens; VI) turbinectomia, em 43 pacientes, média de idade de 33,4 anos (9-70 anos), dos quais 39,6\% eram mulheres e 60,4\%, homens; VII) turbinoplastia, em 55 pacientes, média de idade de 29,3 anos (16-69 anos), dos quais 51\% eram mulheres e 49\%, homens; VIII) outras.

As cirurgias do presente estudo foram realizadas pelos residentes do departamento de otorrinolaringologia sob supervisão do docente da especialidade. Os residentes do segundo ano (R2) realizaram as septoplastias, cauterizações intra-turbinais, turbinectomias e turbinoplastias, ficando as demais cirurgias sob responsabilidade do R3 e do R4 (Tabela 5).

Tabela 3. Principais diagnósticos cirúrgicos encontrados.

\begin{tabular}{lcccc}
\hline Diagnóstico cirúrgico & $\begin{array}{c}\text { Número } \\
\text { de pacientes }\end{array}$ & $\begin{array}{c}\text { Média } \\
\text { de idade }\end{array}$ & $\begin{array}{c}\text { Sexo } \\
\text { Masculino(\%) }\end{array}$ & $\begin{array}{c}\text { Sexo } \\
\text { Feminino(\%) }\end{array}$ \\
\hline Desvio de septo nasal & 457 & 28,1 & 60,6 & 39,4 \\
Deformidadenasal pós-trauma & 287 & 26,7 & 34,5 & 65,5 \\
Hipertrofiade cornetos & 153 & 28,5 & 62,7 & 37,3 \\
Polipose rinossinusal & 73 & 41,3 & 65,7 & 34,3 \\
RinossinusiteCrônica & 32 & 30 & 62,5 & 37,5 \\
\hline
\end{tabular}

Tabela 4. Número de pacientes que realizaram cirurgia isolada ou associada, dentre as mais frequentes.

\begin{tabular}{lcc}
\hline Cirurgia & \multicolumn{2}{c}{ Número de pacientes } \\
& Cirurgiaisolada & Cirurgia associada \\
\hline Septoplastia & 205 & 183 \\
Rinosseptoplastia & 208 & 7 \\
FESS & 79 & 52 \\
Cauterizaçãointra-turbinal & 5 & 109 \\
Rinoplastia Reparadora & 67 & 6 \\
Turbinectomia & 5 & 38 \\
Turbinoplastia & 9 & 46 \\
\hline
\end{tabular}

Tabela 5. Principais cirurgias realizadas.

\begin{tabular}{lcccc}
\hline Cirurgia & $\begin{array}{c}\text { Número } \\
\text { de pacientes }\end{array}$ & $\begin{array}{c}\text { Média } \\
\text { deidade }\end{array}$ & $\begin{array}{c}\text { Sexo } \\
\text { Masculino(\%) }\end{array}$ & $\begin{array}{c}\text { Sexo } \\
\text { Feminino(\%) }\end{array}$ \\
\hline Septoplastia & 388 & 28,2 & 65,2 & 34,8 \\
Rinosseptoplastia & 215 & 27,1 & 37,2 & 62,8 \\
FESS & 131 & 37,3 & 62,5 & 37,5 \\
Cauterizaçãointra-turbinal & 114 & 26,3 & 72 & 28 \\
RinoplastiaReparadora & 73 & 26,6 & 23,3 & 76,7 \\
Turbinectomia & 43 & 33,4 & 60,4 & 39,6 \\
Turbinoplastia & 55 & 29,3 & 49 & 5 I \\
\hline
\end{tabular}


Do total de 33 pacientes com 60 anos ou mais, $72,7 \%$ eram do sexo masculino e $27,3 \%$ do sexo feminino, com média de idade de 66,4 anos (60-80 anos). Os principais diagnósticos cirúrgicos encontrados foram: I) desvio de septo nasal, em 11 pacientes (28,6\%); II) polipose rinossinusal, em 7 pacientes (24\%). Os procedimentos cirúrgicos mais realizados nessa faixa etária foram: I) FESS, realizada em 14 pacientes (42,4\%); e II) septoplastia, em 9 pacientes (27,3\%).

Entre as crianças (menores que 15 anos), encontramos 74 pacientes, sendo $66,2 \%$ do sexo masculino e $33,8 \%$ do sexo feminino. A média de idade foi de 11,08 anos (414 anos). Os principais diagnósticos cirúrgicos encontrados foram: I) desvio de septo nasal, em 47 pacientes $(63,5 \%)$; II) hipertrofia de conchas nasais, em 13 pacientes (17,5\%); III) rinossinusite crônica, em 7 pacientes $(9,5 \%)$. As cirurgias mais realizadas foram: I) septoplastia, em 46 pacientes (62,1\%); FESS, em 17 pacientes (23\%).

\section{DISCUSSÃO}

No Brasil, em 2003, do total de 22.396 cirurgias rinossinusais realizadas no âmbito do SUS, a mais realizada foi à redução de fratura de ossos do nariz ( $28,8 \%$ do total), seguida de septoplastia (25\%), rinoplastia (15,3\%) e turbinectomia (11\%) (4). O quadro é diferente quando comparado com o perfil de uma clínica privada. Se no SUS prevalecem as cirurgias na faringe, com $45,6 \%$ do total, seguidas pelas cirurgias nasais, com 28\% (2), no âmbito privado as cirurgias nasais praticamente se igualam às de faringe, com $34 \%$ e $33 \%$, respectivamente, em recente estudo realizado. Além disso, o principal procedimento rinossinusal foi à turbinectomia, com ou sem septoplastia, representando um terço do total de cirurgias otorrinolaringológicas realizadas (3).

Neste serviço, a septoplastia representou 34\% do total de cirurgias rinossinusais no período, seguida pela rinosseptoplastia (18,9\%), FESS (11,5\%) e cauterização intra-turbinal (10\%). Entre as indicações cirúrgicas, o desvio de septo nasal esteve presente em 52,4\% dos pacientes, seguido pela deformidade nasal pós-trauma $(32,9 \%)$, hipertrofia de cornetos $(17,5 \%)$ e polipose rinossinusal (8,3\%). Diferentemente do volume cirúrgico em clínica privada, em nosso serviço predomina a septoplastia.

Nota-se que $25 \%$ das cirurgias nasais realizadas são consideradas cirurgias estéticas da face (rinosseptoplastia e rinoplastia reparadora), o que demonstra uma importante característica do serviço estudado, preparando, dessa forma, o residente para essa outra modalidade de atuação.
Em relação à septoplastia, procedimento mais realizado neste serviço, foram operados 388 pacientes. A distribuição entre os sexos, com leve predomínio nos homens, e a média de idade, na faixa dos 30 anos, foi semelhante à encontrada em outros trabalhos $(5,6)$. O desvio de septo, que incluiu 457 pacientes, apresentou média de idade de 28,1 anos, sendo $60,6 \%$ do sexo masculino e, 39,4\%, do feminino. Em estudo promovido por Oliveira e cols. (7), que revelou prevalência do desvio de septo em $60,3 \%$ dos transeuntes voluntários avaliados, houve um perfil de gênero diferente deste estudo. Dos 322 pacientes com desvio de septo, cirúrgico ou não, 59\% eram mulheres e $41 \%$, homens.

A segunda cirurgia mais realizada, a rinosseptoplastia, abordou 215 casos. Mais uma vez houve o predomínio de jovens, com média de idade abaixo dos 30 anos. A distribuição entre os sexos foi significativamente maior entre as mulheres $(62,8 \%)$, semelhante ao encontrado por Oropeza e cols. (8), cuja prevalência feminina foi de $56 \%$.

O terceiro procedimento mais frequente foi à cirurgia endoscópica funcional dos seios paranasais (FESS), envolvendo pacientes com maior média de idade $(37,3$ anos). Em outra análise (9), ainda que tenham sido selecionados apenas pacientes com rinossinusite fúngica, podemos observar também faixas etárias médias mais elevadas (média de 40,6 anos). Nesse caso não houve diferença estatística entre os sexos, diferentemente do encontrado, cujo predomínio foi no sexo masculino.

A FESS é empregada em parte dos pacientes com hipertrofia de conchas nasais, polipose nasossinusal, pólipo de Killian, rinossinusite crônica e em outras situações clínico-cirúrgicas.

Avaliando-se os 153 pacientes resistentes a tratamento clínico e que foram operados por hipertrofia de conchas nasais, por FESS ou por outro procedimento, a média de idade foi de 28,5 anos, com predomínio significativo do sexo masculino (62,7\%). Cintra e cols. (10) encontraram média de idade bastante semelhante a este estudo (28 anos), porém sem predominância quanto ao sexo.

A polipose rinossinusal, presente em 73 pacientes, apresentou média de idade de 41,3 anos, compatível com a literatura (11), confirmando ser esta uma doença rara em crianças e adolescentes. Couto e cols. (12) descreveram predomínio do sexo masculino, representando $62,9 \%$ dos indivíduos, muito semelhante ao encontrado neste estudo $(65,7 \%)$.

Em relação aos 32 pacientes com rinossinusite crônica, a média de idade foi de 30 anos, com $62,5 \%$ de homens e 37,5\% de mulheres. Geminiani e cols. (13) 
apontam média de idade de 40 anos e equilíbrio na distribuição entre homens, 51,5\%, e mulheres, 48,5\%.

Quanto ao Pólipo de Killian, dos 10 pacientes analisados, a média de idade foi de 21,3 anos (9-45 anos), $60 \%$ abaixo dos 20 anos, sendo 30\% mulheres e $70 \%$ homens. Franche e cols. (14) apontam média de idade semelhante, 27,5 anos (7-75 anos), mas com maior prevalência no sexo feminino, com 58,6\%. Já Freitas e cols. (15) indicam maior recorrência abaixo dos 20 anos, representando cerca de $70 \%$ dos pacientes estudados, e prevalência no sexo masculino $(1: 1,3)$.

A rinoplastia reparadora, a quinta cirurgia mais prevalente no presente estudo, também se mostra predominante em adultos jovens, porém com uma ampla prevalência no sexo feminino, de acordo com o apresentado por Patrocínio e cols. (16).

Outro procedimento comum na prática diária dos otorrinolaringologistas é a turbinectomia, tratamento eficaz para obstrução nasal secundária à rinite hipertrófica, que no presente estudo, foi prevalente em indivíduos acima dos 30 anos e do sexo masculino, diferentemente do encontrado por BARBosa e cols. (17), cuja média de idade foi de 25 anos, sem predomínio por sexo. No entanto, o estudo referido avalia apenas pacientes que tiveram diagnóstico de rinopatia alérgica, o que poderia explicar essa diferença.

Houveram apenas 11 reduções de fraturas nasais realizadas neste serviço no período analisado ( $82 \%$ no sexo masculino e $18 \%$ no sexo feminino), com uma média de idade de 24,1 anos (variando de 18 a 53 anos), semelhante ao encontrado por MonTovani e cols.(18). Uma das explicações para essa pequena quantidade de procedimentos seria a subnotificação dos traumas nasais, visto que, muitas vezes, a correção dessas fraturas ocorre nas salas de prontoatendimento médico. Vale ressaltar também que, parte das reduções de fratura nasal neste hospital, é realizada por outra especialidade, o que reduz o volume de atendimento para a equipe de otorrinolaringologia.

Destaca-se ainda, 10 pacientes com epistaxe grave no período, com média de idade de 49,5 anos (19-80 anos), com prevalência absoluta no sexo masculino (90\%). SANTOS e cols. (19) apontam também essa média de idade mais elevada para os casos cirúrgicos de epistaxe, com média de 50,9 anos (32-78 anos) e prevalência do sexo masculino, com $67 \%$ dos casos.

\section{CONCLUSÃO}

Conclui-se que o principal diagnóstico cirúrgico rinossinusal realizado neste estudo foi o desvio de septo nasal, sendo a septoplastia a cirurgia mais executada. Os pacientes são em sua maioria adultos jovens e do sexo masculino, tendo recebido, na maior parte das vezes, apenas uma indicação cirúrgica.

Contribui-se, dessa forma, com a escassa produção científica sobre esse tipo de casuística.

\section{REFERÊNCIAS BIBLIOGRÁFICAS}

1. Conselho regional de medicina do estado de São Paulo. Especialidades médicas no estado de São Paulo. Estudos Cremesp - Centro de dados do Cremesp. Disponível em: www.cremesp.org.br

2. Associação Brasileira de Otorrinolaringologia e Cirurgia Cérvico-Facial. Censo 2007 - 2008. Disponível em: http:// www.aborlccf.org.br/imageBank/Censo\%202007-2008.pdf

3. Patrocinio LG, Barreto DM, Rodrigues LF, Patrocinio TG, Coelho SR, Patrocinio JA. Perfil do atendimento otorrinolaringológico em clínica privada. Arq Int Otorrinolaringol. 2007, 11(2):130-34.

4. Gouveia MCL, Lessa FJD, Rodrigues MB, Caldas Neto SS. Perfil de internamento por morbidade otorrinolaringológica com tratamento cirúrgico - Brasil, 2003. Rev Bras Otorrinolaringol. 2005, 71(6):698-04.

5. Caldas Neto S, Oliveira RL, Caldas N. Uso da cola de fibrina na prevenção de sangramento e hematoma pós-operatório em septoplastias. Rev Bras Otorrinolaringol. 2002, 68(5):635-38.

6. Caniello M, Passerotti GH, Goto EY, Voegels RL, Butugan O. Uso de antibióticos em septoplastias: é necessário? Rev Bras Otorrinolaringol. 2005, 71(6):734-38.

7. Oliveira AKP, Elias Junior E, Santos LV, Bettega SG, Mocellin M. Prevalência do desvio de septo nasal em Curitiba, Brasil. Arq Int Otorrinolaringol. 2005, 9(4):288-292.

8. Oropeza FJR, Marin FJS, Chávez MEH. Manejo de la base nasal mediante resección de husos de piel de la columnilla. Acta Otorrinolaringol Esp. 2006, 57:405-11.

9. Dall'Igna C, Palombini BC, Anselmi F, Araújo E, Dall'Igna DP. Rinossinusite fúngica em pacientes com infecção nasossinusal crônica. Rev Bras Otorrinolaringol. 2005, 71(6):712-20.

10. Cintra PPVC, Lima WTA. Comparação das técnicas de turbinectmia com laser de CO2 e laser a diodo. Rev Bras de Otorrinolaringol. 2003, 69(5):612-20. 
11. Souza BB, Serra MF, Dorgam JV, Sarreta SMC, Melo VR, Anselmo-Lima WT. Polipose nasossinusal: doença inflamatória crônica evolutiva? Rev Bras Otorrinolaringol. 2003, 69(3):318-25.

12. Couto LGF, Fernandes AM, Brandão DF, Neto DS, Valera FCP, Anselmo-Lima WT. Aspectos histológicos do pólipo rinossinusal. Rev Bras Otorrinolaringol. 2008, 74(2):20712.

13. Geminiani RJ, Vitale RF, Mazer AB, Gobbo HPC, Silva Neto JJ, Lima JCB. Comparação entre tomografia computadorizada e endoscopia nasal no diagnóstico de rinossinusite crônica. Arq Int Otorrinolaringol. 2007, 11(4):402-05.

14. Franche GLS, Granzotto EH, Borba AT, Hermes F, Saleh CS, Souza PA. Polipectomia endoscópica com meatotomia média como tratamento de pólipo antrocoanal. Rev Bras Otorrinolaringol. 2007, 73(5):689-92.
15. Freitas MR, Giesta RP, Pinheiro SD, Silva VC. Pólipo antrocoanal: revisão de dezesseis casos. Rev Bras Otorrinolaringol. 2006, 72(6):831-35.

16. Patrocínio LG, Patrocínio, JA. Uso de enxertos em rinoplastia. Arq Int Otorrinolaringol. 2001, 5(1):21-5.

17. Barbosa AA, Caldas N, Morais AX, Campos AJC, Caldas S, Lessa F. Avaliação da sintomatologia pré e pós-operatória de pacientes submetidos à turbinectomia inferior. Rev Bras Otorrinolaringol. 2005, 71(4):468-71.

18. Montovani JC, Campos LMP, Gomes MA, Moraes VRS, Ferreira FB, Nogueira EA. Etiologia e incidência das fraturas faciais em adultos e crianças: experiência em 513 casos. Rev Bras Otorrinolaringol. 2006, 72(2):235-41.

19. Santos RP, Leonhardt FD, Ferri RG, Gregório LC. Ligadura endoscópica endonasal da artéria esfenopalatina para epistaxe severa. Rev Bras Otorrinolaringol. 2002, 68(4):511-14. 